\title{
DISCRETE SCHRÖDINGER OPERATORS WITH RANDOM ALLOY-TYPE POTENTIAL
}

\author{
ALEXANDER ELGART, HELGE KRÜGER, MARTIN TAUTENHAHN, \\ AND IVAN VESELIĆ
}

\begin{abstract}
We review recent results on localization for discrete alloytype models based on the multiscale analysis and the fractional moment method, respectively. The discrete alloy-type model is a family of Schrödinger operators $H_{\omega}=-\Delta+V_{\omega}$ on $\ell^{2}\left(\mathbb{Z}^{d}\right)$ where $\Delta$ is the discrete Laplacian and $V_{\omega}$ the multiplication by the function $V_{\omega}(x)=$ $\sum_{k \in \mathbb{Z}^{d}} \omega_{k} u(x-k)$. Here $\omega_{k}, k \in \mathbb{Z}^{d}$, are i.i.d. random variables and $u \in \ell^{1}\left(\mathbb{Z}^{d} ; \mathbb{R}\right)$ is a so-called single-site potential. Since $u$ may change sign, certain properties of $H_{\omega}$ depend in a non-monotone way on the random parameters $\omega_{k}$. This requires new methods at certain stages of the localization proof.
\end{abstract}

\section{Contents}

1. Introduction 2

2. Discrete Anderson models with general alloy-type potential 3

3. Localization properties 4

4. The spectrum 7

5. Averaging of determinants and resolvents 8

5.1. Estimates on polynomials and resolvents 9

5.2. Cartan estimates 10

6. Wegner estimates 11

7. Localization via fractional moment method 13

7.1. Boundedness of fractional moments 13

7.2. Decay of fractional moments 14

7.3. Localization 15

8. Localization via multiscale analysis 18

8.1. Probabilistic estimates 20

8.2. An application of Cartan's lemma 20

References 22

2000 Mathematics Subject Classification. 82B44, 60H25, 35J10.

Key words and phrases. multiscale analysis, fractional moment method, Cartan's Theorem, localization, discrete alloy-type model, non-monotone, sign-indefinite, single-site potential. 


\section{INTRODUCTION}

The paradigmatic model for the study of localization properties of quantum states of single electrons in disordered solids is the Anderson Hamiltonian on the lattice $\mathbb{Z}^{d}$. It consists of the sum of the finite difference Laplacian and a multiplication operator by a sequence of independent identically distributed random variables. There are two independent (though related in sprit) frequently used methods to prove rigorously in appropriate energy and disorder regimes localization statements: the multiscale analysis and the fractional moment method. Both of them rely strongly on the independence property of the random variables. If this property is removed much less is know. Specific cases of random fields with correlations which have been studied so far include the Gaussian field (cf. Section 4 of [DK91] and Section 4 of AM93) and potentials whose distribution is given by a completely analytic Gibbs measure (cf. Section 5 of [DK91] and [DS87]).

A particularly problematic aspect of dependence are negative correlations between values of the potential at different lattice sites. This is intuitively clear when having in mind the role played by the local variation of eigenvalues in localization proofs. An example of a random potential which exhibits negative correlations is an Anderson or alloy-type potential with single-site potentials of changing sign: If one increases a single coupling constant there are regions in space where the potential increases and others where it decreases. Such models have been studied in a number of works devoted to the continuum setting, i.e. for operators on $\mathbb{R}^{d}$. In this paper we want to summarize and discuss a number of results which have been obtained more recently for their lattice counterparts.

The study of Anderson-type models with sign-indefinite single-site potentials can be seen as part of the interest attracted recently by several classes of random operators with a non-monotone dependence on the random variables. They exhibit a variety of intriguing features not encountered in the standard alloy-type model: Already when considering the very basic features of the spectrum as a subset of the real line, one sees that it makes an essential difference whether the operator depends in a monotone or non-monotone way on the random variables entering the model. For operators with monotone parameter dependence the spectral bottom of the operator family corresponds to the configuration where all random variables are set to one of the extremal values. Furthermore, in the monotone situation the band structure of the spectrum can be analyzed using rather basic bracketing arguments, see e.g. KSS98b]. It is consistent with these elementary examples of the advantages of monotonicity that there is a rather good understanding of typical energy/disorder regimes where monotone models exhibit localization of waves, see the monographs and survey articles [Sto01, KM07, Ves07, Kir08].

If the dependence of the operator, respectively the quadratic form, on the random variables is not monotone, already the identification of the spectral minimum is a highly non-trivial question, see e.g. [BLS08, KN09]. For 
more intricate properties, like the regularity of the density of states or the analysis of spectral fluctuation boundaries, the difference between monotone and non-monotone models is even more striking.

Nevertheless there has been a continuous effort to bring the understanding of models with non-monotone dependence on the randomness to a similar level as the one for monotone models. This includes alloy-type Schrödinger operators with single-site potentials of changing sign, see e.g. Klo95, Sto00, Klo02, Ves02, HK02, KV06, KN09, and their lattice counterparts cf. e.g. Ves10b, ETV10, Ves10a, TV10, ETV11, Krü]. Electromagnetic Schrödinger operators with random magnetic field [Uek94, Uek00, HK02, KNNN03, Uek08, Bou09, EHc, EHa, EHb, Laplace-Beltrami operators with random metrics LPV04, LPPV08, LPPV09, as well as the random displacement model, cf. e.g. [Klo93, BLS08, KLNS], are other examples with non-monotonous parameter dependence.

Another relevant model without obvious monotonicity is a random potential given by a Gaussian stochastic field with sign-changing covariance function, c.f. HLMW01, Uek04, Ves11.

The methods which have been developed for the discrete alloy-type potentials with sign-indefinite single-site potentials presented here could be applied to other non-monotone models, as well. In fact the results of [Krü] apply to a much larger class of lattice Schrödinger operator with non-monotone, correlated random potential.

\section{Discrete Anderson models With General Alloy-type POTENTIAL}

To define the random operators we will be looking at, we first introduce the corresponding Hilbert and probability spaces. Let $d \geq 1$. For $x \in \mathbb{Z}^{d}$ we denote by $|x|_{1}=\sum_{i=1}^{d}\left|x_{i}\right|$ and $|x|_{\infty}=\max \left\{\left|x_{1}\right|, \ldots,\left|x_{d}\right|\right\}$ the $\ell^{1}$ and $\ell^{\infty}$ norms on $\mathbb{Z}^{d}$. For $\Gamma \subset \mathbb{Z}^{d}$ we introduce the Hilbert space $\ell^{2}(\Gamma)=\{\psi: \Gamma \rightarrow$ $\left.\mathbb{C}: \sum_{k \in \Gamma}|\psi(k)|^{2}<\infty\right\}$ with inner product $\langle\phi, \psi\rangle=\sum_{k \in \Gamma} \overline{\phi(k)} \psi(k)$.

For each $\Gamma \subset \mathbb{Z}^{d}$ we introduce a probability space $\left(\Omega_{\Gamma}, \mathcal{A}_{\Gamma}, \mathbb{P}_{\Gamma}\right)$. Here $\Omega_{\Gamma}$ is the product $\Omega_{\Gamma}:=\times_{k \in \Gamma} \mathbb{R}, \mathcal{A}_{\Gamma}$ is the associated product sigma algebra generated by cylinder sets, and $\mathbb{P}_{\Gamma}(\mathrm{d} \omega):=\prod_{k \in \Gamma} \mu\left(\mathrm{d} \omega_{k}\right)$ the product measure, with $\mu$ a probability measure on $\mathbb{R}$ with bounded support. The mathematical expectation with respect to $\mathbb{P}_{\Gamma}$ is denoted by $\mathbb{E}_{\Gamma}$. Note that the projections $\Omega \ni \omega=\left\{\omega_{k}\right\}_{k \in \Gamma} \mapsto \omega_{j}, j \in \Gamma$ give rise to a collection of independent identically distributed (i.i.d.) bounded real random variables. If $\Gamma=\mathbb{Z}^{d}$ we will suppress the subscript $\Gamma$ in $\Omega_{\Gamma}, \mathbb{P}_{\Gamma}$ and $\mathbb{E}_{\Gamma}$.

On $\ell^{2}\left(\mathbb{Z}^{d}\right)$ we consider the discrete random Schrödinger operator

$$
H_{\omega}:=-\Delta+\lambda V_{\omega}, \quad \omega \in \Omega, \quad \lambda>0 .
$$


Here $\Delta, V_{\omega}: \ell^{2}\left(\mathbb{Z}^{d}\right) \rightarrow \ell^{2}\left(\mathbb{Z}^{d}\right)$ denote the discrete Laplace and a random multiplication operator defined by

$$
(\Delta \psi)(x):=\sum_{|e|_{1}=1} \psi(x+e) \quad \text { and } \quad\left(V_{\omega} \psi\right)(x):=V_{\omega}(x) \psi(x) .
$$

The parameter $\lambda$ models the strength of the disorder and $\omega$ denotes the randomness. It enters the potential in the following way. Let the single-site potential $u: \mathbb{Z}^{d} \rightarrow \mathbb{R}$ be a function in $\ell^{1}\left(\mathbb{Z}^{d} ; \mathbb{R}\right)$. We assume that the random potential $V_{\omega}$ has an alloy-type structure, i.e. the potential value

$$
V_{\omega}(x):=\sum_{k \in \mathbb{Z}^{d}} \omega_{k} u(x-k)
$$

at a lattice site $x \in \mathbb{Z}^{d}$ is a linear combination of the i.i.d. random variables $\omega_{k}, k \in \mathbb{Z}^{d}$, with coefficients provided by the single-site potential. We assume (without loss of generality) that $0 \in \operatorname{supp} u$. The family of operators $H_{\omega}$, $\omega \in \Omega$, in Eq. (11) is called discrete alloy-type model.

Notice that the single-site potential $u$ may change its sign. As a consequence the quadratic form associated to $H_{\omega}$ does not necessarily depend in a monotone way on the random parameters $\omega_{k}, k \in \mathbb{Z}^{d}$. This is in sharp contrast to the properties of the standard Anderson model which corresponds to the choice of the single-site potential $u=\delta_{0}$. Here

$$
\delta_{k}(j)=\left\{\begin{array}{l}
1 \text { if } k=j, \\
0 \text { else }
\end{array}\right.
$$

denotes the Dirac function.

\section{LOCALIZATION PROPERTIES}

We present several properties related to localization. They concern on the one hand several mathematical signatures of localization, and on the other estimates on the average of resolvents and number of eigenvalues in intervals of finite volume systems, which are instrumental in the arguments leading to localization. They are well established for the standard Anderson model on $\mathbb{Z}^{d}$, see e.g. [Sto01, GK04, Kir08] and the references therein.

Definition 3.1 (Dynamical localization). A selfadjoint operator $H$ on $\ell^{2}\left(\mathbb{Z}^{d}\right)$ is said to exhibit dynamical localization in the interval $I \subset \mathbb{R}$, if for every $x \in \mathbb{Z}^{d}$ and $p \geq 1$ we have

$$
\sup _{t \in \mathbb{R}}\left(\sum_{n \in \mathbb{Z}^{d}}\left(1+|n|_{\infty}\right)^{p}\left|\left\langle\delta_{n}, \mathrm{e}^{-\mathrm{i} t H} \chi_{I}(H) \delta_{x}\right\rangle\right|^{2}\right)<\infty .
$$

Definition 3.2 (Spectral and exponential localization). Let $I \subset \mathbb{R}$. A selfadjoint operator $H: \ell^{2}\left(\mathbb{Z}^{d}\right) \rightarrow \ell^{2}\left(\mathbb{Z}^{d}\right)$ is said to exhibit exponential localization in $I$, if the spectrum of $H$ in $I$ is exclusively of pure point type, i.e. 
$\sigma_{\mathrm{c}}(H) \cap I=\emptyset$, and the eigenfunctions of $H$ corresponding to the eigenvalues in $I$ decay exponentially. If $I=\mathbb{R}$, we say that $H$ exhibits exponential localization.

A family of operators $\left(H_{\omega}\right)_{\omega}$ indexed by elements of a probability space $(\Omega, \mathbb{P})$ is said to exhibit dynamical/exponential localization in the interval $I \subset \mathbb{R}$ if the corresponding property holds for $H_{\omega}$ for almost all $\omega \in \Omega$.

Several important properties of random Hamiltonians are defined in terms of restrictions to a finite system size. We review them next. Let $\Gamma \subset \mathbb{Z}^{d}$. We define $P_{\Gamma}: \ell^{2}\left(\mathbb{Z}^{d}\right) \rightarrow \ell^{2}(\Gamma)$ by

$$
P_{\Gamma} \psi:=\sum_{k \in \Gamma} \psi(k) \delta_{k},
$$

where here $\delta_{k}$ is the Dirac function in $\ell^{2}(\Gamma)$. The restricted operators $\Delta_{\Gamma}, V_{\omega, \Gamma}, H_{\omega, \Gamma}: \ell^{2}(\Gamma) \rightarrow \ell^{2}(\Gamma)$ are defined by

$$
\Delta_{\Gamma}:=P_{\Gamma} \Delta P_{\Gamma}^{*}, \quad V_{\omega, \Gamma}:=P_{\Gamma} V_{\omega} P_{\Gamma}^{*}, \quad H_{\omega, \Gamma}:=P_{\Gamma} H_{\omega} P_{\Gamma}^{*}=-\Delta_{\Gamma}+V_{\omega, \Gamma} .
$$

For $z \in \mathbb{C} \backslash \sigma\left(H_{\omega, \Gamma}\right)$ we define the corresponding resolvent $G_{\omega, \Gamma}(z):=\left(H_{\omega, \Gamma}-\right.$ $z)^{-1}$ and the Green function

$$
G_{\omega, \Gamma}(z ; x, y):=\left\langle\delta_{x},\left(H_{\omega, \Gamma}-z\right)^{-1} \delta_{y}\right\rangle, \quad x, y \in \mathbb{Z}^{d} .
$$

If $\Gamma=\mathbb{Z}^{d}$ we drop the subscript $\Gamma$ in $H_{\omega, \Gamma}, G_{\omega, \Gamma}(z)$ and $G_{\omega, \Gamma}(z ; x, y)$. If $\Lambda \subset \mathbb{Z}^{d}$ is finite, $|\Lambda|$ denotes the number of elements of $\Lambda$. We will use the notation $\mathbb{R}^{+}:=\{x \in \mathbb{R}: x>0\}$.

Definition 3.3 (Decay of fractional moments of the Green function). There exist constants $s \in(0,1)$ and $A, \gamma \in \mathbb{R}^{+}$such that for all $\Gamma \subset \mathbb{Z}^{d}, z \in \mathbb{C} \backslash \mathbb{R}$ and $x, y \in \Gamma$ we have

$$
\mathbb{E}\left\{\left|G_{\omega, \Gamma}(z ; x, y)\right|^{s}\right\} \leq A \mathrm{e}^{-\gamma|x-y|_{\infty}} .
$$

For $x \in \mathbb{Z}^{d}$ and $L>0$, we denote by $\Lambda_{L, x}=\left\{k \in \mathbb{Z}^{d}:|x-k|_{\infty} \leq L\right\}$ the cube of side length $2 L+1$ centred at $x$. For the cube centered at zero we use the notation $\Lambda_{L}=\Lambda_{L, 0}$. We also we write $H_{\omega, L}$ instead of $H_{\omega, \Lambda_{L}}$ and $G_{\omega, L}(z)$ and $G_{\omega, L}(z ; x, y)$ instead of $G_{\omega, \Lambda_{L}}(z)$ and $G_{\omega, \Lambda_{L}}(z ; x, y)$. For $\Lambda \subset \mathbb{Z}^{d}$ we denote by $\partial^{\mathrm{i}} \Lambda=\left\{k \in \Lambda: \#\left\{j \in \Lambda:|k-j|_{1}=1\right\}<2 d\right\}$ the interior boundary of $\Lambda$ and by $\partial^{\mathrm{o}} \Lambda=\partial^{\mathrm{i}} \Lambda^{\mathrm{c}}$ the exterior boundary of $\Lambda$. Here $\Lambda^{\mathrm{c}}=\mathbb{Z}^{d} \backslash \Lambda$ denotes the complement of $\Lambda$.

Definition 3.4 (Wegner estimate). There are constants $C_{\mathrm{W}}, L_{0} \in \mathbb{R}^{+}, b \geq$ 1 , and a function $f: \mathbb{R}^{+} \rightarrow \mathbb{R}^{+}$satisfying $\lim _{\varepsilon \backslash 0} f(\varepsilon)=0$ such that we have for any $L \geq L_{0}, E \in \mathbb{R}$ and $\varepsilon \in(0,1)$

$$
\begin{aligned}
\mathbb{P}\left\{[E-\varepsilon, E+\varepsilon] \cap \sigma\left(H_{\omega, L}\right) \neq \emptyset\right\} & \leq \mathbb{E}\left\{\operatorname{Tr}\left[\chi_{[E-\varepsilon, E+\varepsilon]}\left(H_{\omega, L}\right)\right]\right\} \\
& \leq C_{\mathrm{W}} f(\varepsilon)(2 L+1)^{b d} .
\end{aligned}
$$

In specific applications of a Wegner estimate (for example as an ingredient for the multiscale analysis) one needs a specific rate of decay on the function $f$ near zero. The original estimate of Wegner Weg81 corresponds to $f(\varepsilon)=$ 
$\varepsilon$ and $b=1$ and implies the Lipschitz-continuity of the integrated density of states. In many situations one can establish (2) with $b=1$ or $b=2$ and $f(\varepsilon)=\varepsilon^{a}$ for $a \in(0,1)$. In certain situations variants of the Wegner estimate which do not control only global property $d\left(E, \sigma\left(H_{\omega, L}\right) \leq \varepsilon\right.$ but also specific coefficients of the resolvent, as well, need to be used, cf. Section 8.2 or [Bou09, Krü].

Definition 3.5. Let $m, L>0, x \in \mathbb{Z}^{d}$ and $E \in \mathbb{R}$. A cube $\Lambda_{L, x}$ is called $(m, E)$-regular (for a fixed potential), if $E \notin \sigma\left(H_{\Lambda_{L, x}}\right)$ and

$$
\sup _{w \in \partial^{\mathrm{i}} \Lambda_{L, x}}\left|G_{\Lambda_{L, x}}(E ; x, w)\right| \leq \mathrm{e}^{-m L} .
$$

Otherwise we say that $\Lambda_{L, x}$ is $(m, E)$-singular.

Rather than looking at the fractional moments of the Green function one can consider the probability of a box to be regular. The decay of these probabilities is closely related to the localization phenomenon. For simplicity we define one variant of this decay property and restrict ourselves to the case of finitely supported single-site potentials $u$.

Definition 3.6 (Probabilistic decay of Green's function). Let $\Theta:=\operatorname{supp} u$ be finite, $I \subset \mathbb{R}$ be an interval and let $p>d, L_{0}>1, \alpha \in(1,2 p / d)$ and $m>0$. Set $L_{k}=L_{k-1}^{\alpha}$, for $k \in \mathbb{N}$. For any $k \in \mathbb{N}_{0}$

$$
\mathbb{P}\left\{\forall E \in I \text { either } \Lambda_{L_{k}, x} \text { or } \Lambda_{L_{k}, y} \text { is }(m, E) \text {-regular }\right\} \geq 1-L_{k}^{-2 p}
$$

for any $x, y \in \mathbb{Z}^{d}$ with $|x-y|_{\infty} \geq 2 L_{k}+\operatorname{diam} \Theta+1$.

Here we denote for finite $\Gamma \subset \mathbb{Z}^{d}$ by diam $\Gamma$ the diameter of $\Gamma$ with respect to the supremum norm, i.e. $\operatorname{diam} \Gamma=\sup _{x, y \in \Gamma}|x-y|_{\infty}$. In the subsequent sections we describe which of these localization properties have been proven for the non-monotone model we are interested in.

The most general result concerning (large disorder) localization for the discrete alloy-type model is [Krü]. Krüger proves for exponentially decaying single-site potentials dynamical localization in the case of sufficiently large disorder. Indeed, this result applies for a class of models including the discrete alloy-type model with exponentially decaying singe-site potential as a special case. Notice that dynamical localization implies spectral localization via the RAGE-Theorem, see e.g. [Sto10], but not vice versa as examples in dRJLS96 show. The proof of Krüger's result uses the multiscale analysis and is discussed in Section 8 .

There are also localization results not using the multiscale analysis but the fractional moment method. In [ETV10] the authors prove exponential localization in the case of space dimension $d=1$, compactly supported singlesite potentials and sufficiently large disorder. This result was extended in [ETV11] to arbitrary space dimension assuming that the single-site potential has fixed sign at the boundary of its support, a property which can be assumed without loss of generality in $d=1$. This result is discussed in Section 7 . 
In Section 5 we present certain estimates concerning averages of polynomials and resolvents which are fundamental for the results presented in Sections 7 and 8 .

In Section 6 we discuss certain results on Wegner estimates for the discrete alloy-type model [Ves10a, PTV11. With the help of such Wegner-estimates one can implement a proof of localization via the multiscale analysis in the regime where an appropriate initial length scale estimate is available.

In the following Section 4 we show that the almost sure spectrum of the discrete alloy-type model is an interval.

\section{The SPECTRum}

Before studying the properties of the spectral measure under the Lebesgue decomposition, one wants to understand basic features of the set $\Sigma \subset \mathbb{R}$ which coincides with the spectrum of $H_{\omega}$ almost surely. This concerns in particular the infimum and supremum of the spectrum and internal spectral edges (if any). For the standard Anderson model there is a nice formula for the spectrum:

$$
\Sigma=[-2 d, 2 d]+\operatorname{supp} \mu
$$

where $[-2 d, 2 d]$ is the spectrum of the free Laplacian $\Delta$ and supp $\mu$ the spectrum of the multiplication operator given by the random potential. Related descriptions of $\Sigma$ for the (continuum) alloy-type model have been studied in KM82. In particular, a description of $\Sigma$ in terms of admissible potentials was given. In many cases this class consists of an appropriate family of periodic potentials. Let us quote a specific result from [KSS98b]: If $S:=\operatorname{supp} \mu$ is a bounded interval and the single-site potential $u$ is non negative, then

$$
\Sigma=\bigcup_{\kappa \in S} \sigma\left(-\Delta+\kappa \sum_{n \in \mathbb{Z}^{d}} u(\cdot-n)\right) .
$$

The proof of this equality uses that $u$ has fixed sign and is thus not applicable in our case. Our result about the set $\Sigma$ is

Theorem 4.1. Let $H_{\omega}$ be a discrete random Schrödinger operators as in (1). If $S:=\operatorname{supp} \mu$ is bounded and connected, then the spectrum of $H_{\omega}$ is almost surely an interval.

Proof. Denote by $\Sigma$ the almost sure spectrum of $H_{\omega}$. It is well know that

$$
\Sigma=\bigcup_{\omega \in S^{\mathbb{Z}^{d}}} \sigma\left(H_{\omega}\right)
$$

cf. the discussion in Section 6 of [Kir89]. Now we assume $0 \in S$ without loss of generality. For $\omega \in S^{\mathbb{Z}^{d}}$ and $t \in[0,1]$ denote $t \cdot \omega=\left(t \omega_{x}\right)_{x \in \mathbb{Z}^{d}}$ the scaled configuration. We then have that $\sigma\left(H_{0 \cdot \omega}\right)=[-2 d, 2 d]$. Fix now $\omega \in S^{\mathbb{Z}^{d}}$. We show that

$$
\Sigma_{\omega}=\bigcup_{t \in[0,1]} \sigma\left(H_{t \cdot \omega}\right)
$$


is an interval. For this purpose note that $\sigma\left(H_{0 \cdot \omega}\right)=[-2 d, 2 d]$ and that the spectral maximum and spectral minimum

$$
[0,1] \ni t \mapsto \max \sigma\left(H_{t \cdot \omega}\right), \quad[0,1] \ni t \mapsto \min \sigma\left(H_{t \cdot \omega}\right)
$$

are continuous functions of $t$. This follows by the min-max-principle. Thus

$$
\Sigma_{\omega}=\left[\min _{t \in[0,1]} \min \sigma\left(H_{t \cdot \omega}\right), \max _{t \in[0,1]} \max \sigma\left(H_{t \cdot \omega}\right)\right] \supset[-2 d, 2 d] .
$$

Choose now $\lambda \in \bigcup_{\omega \in S^{\mathbb{Z}^{d}}} \sigma\left(H_{\omega}\right)$. Then there exists $\tilde{\omega} \in S^{\mathbb{Z}^{d}}$ such that $\lambda \in$ $\sigma\left(H_{\tilde{\omega}}\right) \subset \Sigma_{\tilde{\omega}}$. Since the latter set is an interval containing $[-2 d, 2 d]$, it follows that $\Sigma$ is an interval.

\section{Averaging of Determinants And Resolvents}

In the energy regime where localization holds, eigenvalues are sensitive to fluctuations of the random potential. In particular, the mathematical expectation leads to a regularization of the finite volume eigenvalue counting function. Likewise, (appropriate) averages of the resolvent enjoy boundedness properties which are impossible to hold for resolvent associated to individual realizations of the random potential. In this section we discuss bounds of the type indicated above and in the following sections their relation to localization proofs.

We start with a well known weak $L^{1}$-bound formulated in Lemma 5.1 (see e.g. $\left[\mathrm{AEN}^{+} 06\right.$, Proposition 3.1] for a more general result), which can be used to obtain bounds on certain averages of resolvents in the monotone case, i.e. where the single-site potential $u$ is non-negative. Recall, a densely defined operator $T$ on some Hilbert space $\mathcal{H}$ with inner product $\langle\cdot, \cdot\rangle_{\mathcal{H}}$ is called dissipative if $\operatorname{Im}\langle x, T x\rangle_{\mathcal{H}} \geq 0$ for all $x \in D(T)$.

Lemma 5.1. Let $A \in \mathbb{C}^{n \times n}$ be a dissipative matrix, $V \in \mathbb{R}^{n \times n}$ diagonal and strictly positive definite and $M_{1}, M_{2} \in \mathbb{C}^{n \times n}$ be arbitrary matrices. Then there exists a constant $C_{\mathrm{W}}$ (independent of $A, V, M_{1}, M_{2}$ and $n$ ), such that

$\mathcal{L}\left\{r \in \mathbb{R}:\left\|M_{1}(A+r V)^{-1} M_{2}\right\|_{\mathrm{HS}}>t\right\} \leq C_{\mathrm{W}}\left\|M_{1} V^{-1 / 2}\right\|_{\mathrm{HS}}\left\|M_{2} V^{-1 / 2}\right\|_{\mathrm{HS}} \frac{1}{t}$.

Here, $\mathcal{L}$ denotes the Lebesgue-measure and $\|\cdot\|_{\mathrm{HS}}$ the Hilbert Schmidt norm.

If the single-site potential $u$ has fixed sign (and compact support) Lemma 5.1 is applicable and yields (together with a decoupling argument) the decay of fractional moments of the Green function. A generalization of Lemma 5.1 also applies to the continuous alloy-type model on $L^{2}\left(\mathbb{R}^{d}\right)$ to obtain bounds on fractional moments of the Green function, see [AEN $\left.{ }^{+} 06\right]$.

Since we allow the single-site potential to change its sign, we want to get rid of the positivity assumption on the operator $V$. The first observation is, that if one considers averages of determinants, then the definiteness of $V$ plays no longer a role. 
5.1. Estimates on polynomials and resolvents. In this section, we discuss estimates as used by Elgart, Tautenhahn, and Veselić in ETV10 and ETV11.

Lemma 5.2 ([ETV10]). Let $n \in \mathbb{N}, P(x)=x^{n}+\ldots$ a polynomial of degree $n$, and $0 \leq \rho \in L^{1}(\mathbb{R}) \cap L^{\infty}(\mathbb{R})$ and $s \in(0,1)$. Then we have

$$
\int_{\mathbb{R}} \frac{1}{|P(x)|^{s / n}} \rho(x) d x \leq\|\rho\|_{L^{1}}^{1-s}\|\rho\|_{\infty}^{s} \frac{2^{s} s^{-s}}{1-s} .
$$

In [ETV10], this result is stated as

$$
\begin{aligned}
\int_{\mathbb{R}}|\operatorname{det}(A+r V)|^{-s / n} \rho(r) \mathrm{d} r & \leq|\operatorname{det} V|^{-s / n}\|\rho\|_{L^{1}}^{1-s}\|\rho\|_{\infty}^{s} \frac{2^{s} s^{-s}}{1-s} \\
& \leq|\operatorname{det} V|^{-s / n}\left(\lambda^{-s}\|\rho\|_{L^{1}}+\frac{2 \lambda^{1-s}}{1-s}\|\rho\|_{\infty}\right)
\end{aligned}
$$

holds for $\lambda>0, A \in \mathbb{C}^{n \times n}$, and $V \in \mathbb{C}^{n \times n}$ invertible. Since $r \mapsto \frac{1}{\operatorname{det}(V)} \operatorname{det}(A+$ $r V$ ) is a monic polynomial of degree $n$, (3) implies this statement. For the converse, use that $P(x)=x^{n}+\sum_{j=0}^{n-1} \alpha_{j} x^{j}$ can be rewritten in terms of the companion matrix as

$$
P(x)=\operatorname{det}(x+A), \quad A=\left(\begin{array}{ccccc}
0 & 0 & \ldots & 0 & -\alpha_{0} \\
1 & 0 & \ldots & 0 & -\alpha_{1} \\
\vdots & \vdots & \ddots & \vdots & \vdots \\
0 & 0 & \ldots & 0 & -\alpha_{n-1} \\
0 & 0 & \ldots & 1 & -\alpha_{n}
\end{array}\right)
$$

The given form of Lemma 5.2 has the advantage that its relation to Pólya's inequality becomes more apparent. Namely, that for any polynomial $P(x)=$ $x^{n}+\ldots$ of degree $n$, we have

$$
|\{x \in \mathbb{R}: \quad|P(x)| \leq \alpha\}| \leq 4\left(\frac{\alpha}{2}\right)^{\frac{1}{n}}
$$

for $\alpha>0$.

In $d=1$ the bound from Lemma 5.2 is precisely what is needed to show the boundedness of averaged fractional powers of the Green function, since certain matrix elements of the Green function can be represented as an inverse of a determinant of the above type. More precisely, if $d=1$ and $\operatorname{supp} u=\{0,1, \ldots, n-1\}$ then we have for all $x \in \mathbb{Z}$ and $z \in \mathbb{C} \backslash \mathbb{R}$

$$
\left|G_{\omega}(z ; x, x+n-1)\right|=\frac{1}{\left|\operatorname{det}\left(A+\omega_{x} \lambda V\right)\right|},
$$

where $V \in \mathbb{R}^{n \times n}$ is diagonal with diagonal elements $u(k-x), k=x, \ldots, x+$ $n-1$, and where $A \in \mathbb{C}^{n \times n}$ is independent of $\omega_{x}$. By Lemma 5.2 one obtains a bound on the expectation of an averaged fractional power on certain Green's function elements, which is sufficient to start the proof of localization via the fractional moment method. See ETV10] for details. 
Let us turn to the higher dimensional case. For $B \in \mathbb{C}^{n \times n}$ we have

$$
\left\|B^{-1}\right\| \leq \frac{\|B\|^{n-1}}{|\operatorname{det}(B)|}
$$

Thus one can use (3) to obtain bounds on the inverse of $A+r V$. More precisely, we have for $s \in(0,1), R>0$ and $A, V$ as above the estimate

$$
\int_{-R}^{R}\left\|(A+r V)^{-1}\right\|^{s / n} \mathrm{~d} r \leq \frac{2 R^{1-s}(\|A\|+R\|V\|)^{s(n-1) / n}}{s^{s}(1-s)|\operatorname{det} V|^{s / n}} .
$$

In $d>1$ Lemma 5.2 is no longer applicable, but the estimate (5) is. However, the problem with the estimate (5) is that the upper bound depends on the background operator $A$. Note that $A$ arises by a Schur-complement formula and has a complicated dependence on the randomness. For this reason we have to assume additional properties on the single-site potential $u$ (i.e. that $u$ has fixed sign on the boundary of its (bounded) support) to show bounds on averaged fractional powers of the Green function, see Section 7 or [ETV11] for details.

5.2. Cartan estimates. We have now discussed the facts from complex analysis on which ETV10 and ETV11 are based. Let us now discuss the ones used in Krï] and Bourgain Bou09, which are based on ideas from quasi-periodic Schroedinger operators see Bourgain, Goldstein and Schlag [BGS02, Bou04, Bou07]

Like the estimates discussed in the first part of this section, they control the size of the set where an analytic function is small. The main difference is that instead of requiring as input that the analytic function is a polynomial of small degree, one assumes that the analytic function is not small at a single point. Here is the simplest form of such an estimate.

Theorem 5.3. Let $f$ be an analytic function on the disc of radius $2 \mathrm{e}$ satisfying

$$
\sup _{|z|<2 \mathrm{e}}|f(z)| \leq 1, \quad|f(0)| \geq \varepsilon .
$$

Then for $s>0$, we have

$$
\left|\left\{x \in[-1,1]: \quad|f(x)| \leq \mathrm{e}^{-s}\right\}\right| \leq 30 \mathrm{e}^{3} \exp \left(-\frac{s}{\log \left(\varepsilon^{-1}\right)}\right) .
$$

Proof. For a proof see Theorem 11.3.4 in [Lev96]. See also Theorem 10.2 in [Krï] for the deduction of this statement.

This form of the Cartan estimate is not sufficient for the application to random Schrödinger operators as done in Bou09] and Krü]. For these results, one needs to apply the Cartan estimate to functions depending on many variables, and thus needs an estimate that is well behaved in the number of variables. Such an estimate was first proven by Nazarov, Sodin, and Volberg [NSV03]. Unfortunately, they work on balls and not on polydisks as necessary for our applications. The result for poly-disks was proven 
by Bourgain in Bou09. We will state here a formulation which was given in Krü].

Theorem 5.4 (Theorem 10.1, [Krï]). Denote by $\mathbb{D}_{r}$ the disc of radius $r$ in $\mathbb{C}$. Let $f:\left(\mathbb{D}_{2 \mathrm{e}}\right)^{n} \rightarrow \mathbb{C}$ be an analytic function satisfying

$$
\|f\|_{L^{\infty}\left(\left(\mathbb{D}_{2 \mathrm{e}}\right)^{n}\right)} \leq 1, \quad|f(0)| \geq \varepsilon .
$$

Then for $s>0$, we have

$$
\frac{\left|\left\{x \in[-1,1]^{n}: \quad|f(x)| \leq \mathrm{e}^{-s}\right\}\right|}{2^{n}} \leq 30 \mathrm{e}^{3} n \exp \left(-\frac{s}{\log \left(\varepsilon^{-1}\right)}\right) .
$$

Note that the dimension dependence is $n$. The proof is based on a clever change to spherical coordinates. We give an exposition on how to apply this to Schrödinger operators in Section 8.2 .

\section{WEGNER ESTIMATES}

This section is concerned with averaging of spectral projections. For any $\omega \in \Omega$ and $L \in \mathbb{N}$ the restriction $H_{\omega, L}$ is a selfadjoint finite rank operator. In particular its spectrum consists entirely of real eigenvalues $E(\omega, L, 1) \leq$ $E(\omega, L, 2) \leq \cdots \leq E\left(\omega, L, \sharp \Lambda_{L}\right)$ counted including multiplicities. Wegner estimates Weg81 are bounds on the expected number of eigenvalues of the finite box Hamiltonians $H_{\omega, L}$ in a compact energy interval $I=[E-\varepsilon, E+\varepsilon]$. They can be used as an ingredient for a localization proof via the multiscale analysis. The symbol $\chi_{I}\left(H_{\omega, L}\right)$ denotes the spectral projection onto $I$ with respect to the operator $H_{\omega, L}$.

Theorem 6.1 (Ves10a,, PTV11]). Assume that $\mu$ has a density $\rho$ of finite total variation and $u$ is not identically zero.

(a) Assume that the single-site potential $u$ has support in $[0, n]^{d} \cap \mathbb{Z}^{d}$. Then there exists a constant $c_{u}$ depending only on $u$ such that for any $L \in \mathbb{N}$, $E \in \mathbb{R}$ and $\varepsilon>0$ we have

$$
\mathbb{E}\left\{\operatorname{Tr} \chi_{[E-\varepsilon, E+\varepsilon]}\left(H_{\omega, L}\right)\right\} \leq c_{u}|\operatorname{supp} u|\|\rho\|_{\mathrm{BV}} \varepsilon(2 L+n)^{d \cdot(n+1)}
$$

(b) Assume $\bar{u}=\sum_{k \in \mathbb{Z}^{d}} u(k) \neq 0$ and that $\rho$ has compact support. Then we have for any $L \in \mathbb{N}, E \in \mathbb{R}$ and $\varepsilon>0$

$$
\mathbb{E}\left\{\operatorname{Tr} \chi_{[E-\varepsilon, E+\varepsilon]}\left(H_{\omega, L}\right)\right\} \leq \frac{8}{\bar{u}} \min \left((2 L)^{d},|\operatorname{supp} u|\right)\|\rho\|_{\mathrm{BV}} \varepsilon(2 L+m)^{d},
$$

where $m \in \mathbb{N}$ is such that $\sum_{\|k\| \geq m}|u(k)| \leq|\bar{u} / 2|$.

(c) Assume there are constants $C, \alpha \in \mathbb{R}^{+}$such that $|u(k)| \leq C e^{-\alpha\|k\|_{1}}$ for all $k \in \mathbb{Z}^{d}$, and that $\rho$ has bounded support. Then there exists $c_{u}>0$ and $I_{0} \in \mathbb{N}_{0}^{d}$ both depending only on $u$ such that for any $L \in \mathbb{N}, E \in \mathbb{R}$ and $\varepsilon>0$ we have

$$
\mathbb{E}\left\{\operatorname{Tr} \chi_{[E-\varepsilon, E+\varepsilon]}\left(H_{\omega, L}\right)\right\} \leq c_{u}\|\rho\|_{\mathrm{BV}} \varepsilon(2 L+1)^{2 d+\left|I_{0}\right|} .
$$

In the case where the support of $u$ is compact, part (b) of Theorem 6.1 has an important corollary. 
Corollary 6.2 ([Ves10a $)$. Assume $\bar{u} \neq 0$ and $\operatorname{supp} u \subset[0, n] \cap \mathbb{Z}^{d}$. Then we have for any $L \in \mathbb{N}, E \in \mathbb{R}$ and $\varepsilon>0$

$$
\mathbb{E}\left\{\operatorname{Tr} \chi_{[E-\varepsilon, E+\varepsilon]}\left(H_{\omega, L}\right)\right\} \leq \frac{4}{\bar{u}} \operatorname{rank} u\|\rho\|_{B V} \varepsilon(2 L+n)^{d} .
$$

In particular, the function $\mathbb{R} \ni E \rightarrow \mathbb{E}\left\{\operatorname{Tr}\left[\chi_{(-\infty, E]}\left(H_{\omega, L}\right)\right]\right\}$ is Lipschitz continuous.

Remark 6.3. (i) Note, that apart of the exponential decay condition on $u$, Theorem 6.1 (c) gives a Wegner estimate for the discrete alloy-type model without any further assumption on the single-site potential. In particular, $u$ may change its sign arbitrarily.

(ii) The proof of Theorem 6.1 is (roughly speaking) based on a transformation of the probability space to recover monotonicity. With other words, once you find a finite linear combination of translated single-site potentials which is positive, then monotone spectral averaging leads to a Wegner estimate, see [KV06, Ves10b, Ves10a, PTV11] where this approach is used.

Remark 6.4 (Continuous alloy-type model). The alloy-type model is the Schrödinger operator $H_{\omega}=-\Delta+V_{0}+V_{\omega}$ on $L^{2}\left(\mathbb{R}^{d}\right)$, where $\Delta$ is the Laplacian on $\mathbb{R}^{d}, V_{0}$ a $\mathbb{Z}^{d}$ periodic potential, and $V_{\omega}$ given by

$$
V_{\omega}(x)=\sum_{k \in \mathbb{Z}^{d}} \omega_{k} U(x-k)
$$

with $U: \mathbb{R}^{d} \rightarrow \mathbb{R}$ a single-site potential. It is assumed that $V_{0}$ and $V_{\omega}$ are infinitesimally bounded with respect to $\Delta$, with constants uniformly bounded in $\omega \in \Omega$. We will be concerned with the case that the distribution $\mu$ has a density $\rho$ of finite total variation. and $U$ is a generalized step-function, i.e.

$$
U(x)=\sum_{k \in \mathbb{Z}^{d}} u(k) w(x-k) .
$$

Here $L_{\mathrm{c}}^{p}\left(\mathbb{R}^{d}\right) \ni w \geq \kappa \chi_{(-1 / 2,1 / 2)^{d}}$ with $\kappa>0, p=2$ for $d \leq 3$ and $p>d / 2$ for $d \geq 4$, and $u \in \ell^{1}\left(\mathbb{Z}^{d} ; \mathbb{R}\right)$ the discrete single-site potential.

In [PTV11] a Wegner estimate similar to part (c) in Theorem6.1] is proven for the (continuous) alloy-type model. More precisely, assume that $U$ is a generalized step-function and there are constants $C, \alpha \in \mathbb{R}^{+}$such that $|u(k)| \leq C \mathrm{e}^{-\alpha\|k\|_{1}}$. Then there exists $c_{U}>0$ and $I_{0} \in \mathbb{N}_{0}^{d}$ both depending only on $U$ such that for any $L \in \mathbb{N}$ and any bounded interval $I:=\left[E_{1}, E_{2}\right] \subset$ $\mathbb{R}$ we have

$$
\mathbb{E}\left\{\operatorname{Tr} \chi_{I}\left(H_{\omega, L}\right)\right\} \leq \mathrm{e}^{E_{2}} c_{U}\|\rho\|_{\operatorname{Var}}|I|(2 l+1)^{2 d+\left|I_{0}\right|} .
$$

Here $H_{\omega, L}$ denotes the restriction of $H_{\omega}$ to the cube $(-L, L)^{d} \subset \mathbb{R}^{d}$ with either Dirichlet or periodic boundary conditions. The stated Wegner estimate is valid for both types of boundary conditions. 
A drawback of this results is that they fail if $u$ is not a generalized step function. Contrary to this, the papers [Klo95, HK02] obtain Wegner estimates for energies in a neighborhood of the infimum of the spectrum which are valid for arbitrary non-vanishing single-site potentials $u \in C_{\mathrm{c}}\left(\mathbb{R}^{d}\right)$ and coupling constants whose distribution has a piecewise absolutely continuous density.

Remark 6.5 (Localization). Notice that the Wegner estimates from Theorem 6.1 are valid on the whole energy axis. Therefore, one can prove localization via multiscale analysis [FS83, DK89] in any energy region where an initial length scale estimate holds. If the single-site potential does not have compact support one has to use a modified version of the multiscale analysis KSS98a.

\section{LOCALIZATION VIA FRACTIONAL MOMENT METHOD}

7.1. Boundedness of fractional moments. The lemmata from Section 5 can be used to obtain bounds on averaged fractional powers of the Green function. One possible approach to overcome the problems arising because of the lack of monotonicity is to use a special transformation of the probability space to recover some monotonicity which makes Lemma 5.1 applicable. This was done in [ETV11, Appendix] to obtain the following Theorem.

Theorem 7.1 ([ETV11]). Assume

(i) The measure $\mu$ has a density $\rho$ in the Sobolev space $W^{1,1}(\mathbb{R})$.

(ii) The single-site potential $u$ has compact support and satisfies $\bar{u}:=$ $\sum_{k \in \mathbb{Z}^{d}} u(k) \neq 0$.

Let further $\Lambda \subset \mathbb{Z}^{d}$ finite and $s \in(0,1)$. Then we have for all $x, y \in \Lambda$ and $z \in \mathbb{C} \backslash \mathbb{R}$

$$
\mathbb{E}\left\{\left|G_{\omega, \Lambda}(z ; x, y)\right|^{s}\right\} \leq \frac{1}{1-s}\left(\frac{2\left\|\rho^{\prime}\right\|_{L^{1}} C}{\bar{u}}\right)^{s} \frac{1}{\lambda^{s}}
$$

where $C$ is a constants depending only on $u$.

The disadvantage of Theorem 7.1 is that it is non-local in the sense that we have to average with respect of the entire disorder present in the model. At the moment we do not know how to conclude the decay of fractional moments of the Green function (cf. Definition 3.3) from the non-local a priori bound in Theorem 7.1. A somewhat stronger condition, however, is sufficient to ensure decay of fractional moments. We will review this result next.

Assumption (A). Assume that

(1) the measure $\mu$ has a bounded, compactly supported density $\rho \in$ $L^{\infty}(\mathbb{R})$.

(2) $\Theta:=\operatorname{supp} u$ is finite and $u(k)>0$ for $k \in \partial^{\mathrm{i}} \Theta$. 
Under Assumption (A) and with the help of Ineq. (5) it is possible to prove a local a priori bound, which is applicable to conclude the decay of fractional moments of the Green function, see Section 7.2. Let us first introduce some more notation. For $x \in \mathbb{Z}^{d}$ we denote by $\mathcal{N}(x)=\left\{k \in \mathbb{Z}^{d}:|x-k|_{1}=1\right\}$ the neighborhood of $x$. For $\Lambda \subset \mathbb{Z}^{d}$ we also define $\Lambda_{x}=\Lambda+x=\left\{k \in \mathbb{Z}^{d}\right.$ : $k-x \in \Lambda\}$.

Lemma 7.2 ([ETV11]). Let Assumption $(A)$ be satisfied, $\Gamma \subset \mathbb{Z}^{d}, m>0$ and $s \in(0,1)$.

(a) Then there is a constant $C_{s}$, depending only on $d, \rho, u, m$ and $s$, such that for all $z \in \mathbb{C} \backslash \mathbb{R}$ with $|z| \leq m$, all $x, y \in \Gamma$ and all $b_{x}, b_{y} \in \mathbb{Z}^{d}$ with $x \in \Theta_{b_{x}}$ and $y \in \Theta_{b_{y}}$

$$
\mathbb{E}_{N}\left\{\left|G_{\omega, \Gamma}(z ; x, y)\right|^{s /(2|\Theta|)}\right\} \leq C_{s} \Xi_{s}(\lambda)
$$

where $\Xi_{s}(\lambda)=\max \left\{\lambda^{-s /(2|\Theta|)}, \lambda^{-2 s}\right\}$ and $N=\left\{b_{x}, b_{y}\right\} \cup \mathcal{N}\left(b_{x}\right) \cup \mathcal{N}\left(b_{y}\right)$.

(b) Then there is a constant $D_{s}$, depending only on $d, \rho, u$ and $s$, such that for all $z \in \mathbb{C} \backslash \mathbb{R}$, all $x, y \in \Gamma$ and all $b_{x}, b_{y} \in \mathbb{Z}^{d}$ with

$$
x \in \Theta_{b_{x}} \cap \Gamma \subset \partial^{\mathrm{i}} \Theta_{b_{x}} \quad \text { and } \quad y \in \Theta_{b_{y}} \cap \Gamma \subset \partial^{\mathrm{i}} \Theta_{b_{y}}
$$

we have

$$
\mathbb{E}_{\left\{b_{x}, b_{y}\right\}}\left\{\left|G_{\omega, \Gamma}(z ; x, y)\right|^{s}\right\} \leq D_{s} \lambda^{-s}
$$

7.2. Decay of fractional moments. Now we explain how the so called finite volume criterion implies exponential decay of the Green function. Together with the a-priori bound from Lemma 7.2 this gives us Theorem 7.5 . For proofs we refer the reader to [ETV11].

To formulate the results of this section we will need the following notation: Let $\Gamma \subset \mathbb{Z}^{d}$, fix $L \geq \operatorname{diam} \Theta+2$, let

$$
B=\partial^{\mathrm{i}} \Lambda_{L}
$$

and define the sets

$$
\hat{\Lambda}_{x}=\left\{k \in \Gamma: k \in \Theta_{b} \text { for some } b \in \Lambda_{L, x}\right\}
$$

and

$$
\hat{W}_{x}=\left\{k \in \Gamma: k \in \Theta_{b} \text { for some } b \in B_{x}\right\} .
$$

Recall that for $\Gamma \subset \mathbb{Z}^{d}$ we denote by $\Gamma_{x}=\Gamma+x=\left\{k \in \mathbb{Z}^{d}: k-x \in \Gamma\right\}$ the translate of $\Gamma$. Hence $\left(\Lambda_{L}\right)_{x}=\Lambda_{L, x}$ and $\hat{W}_{x}$ is the union of translates of $\Theta$ along the sides of $B_{x}$, restricted to the set $\Gamma$. For $\Gamma \subset \mathbb{Z}^{d}$ we can now introduce the sets

$$
\Lambda_{x}:=\hat{\Lambda}_{x}^{+} \cap \Gamma \quad \text { and } \quad W_{x}=\hat{W}_{x}^{+} \cap \Gamma
$$

which will play a role in the assertions below. 
Theorem 7.3 (ETV11], Finite volume criterion). Suppose that Assumption $(A)$ is satisfied. Let $\Gamma \subset \mathbb{Z}^{d}, z \in \mathbb{C} \backslash \mathbb{R}$ with $|z| \leq m$ and $s \in(0,1 / 3)$. Then there exists a constant $B_{s}$ which depends only on $d, \rho, u, m, s$, such that if the condition

$$
b_{s}(\lambda, L, \Lambda):=\frac{B_{s} L^{3(d-1)} \Xi_{s}(\lambda)}{\lambda^{2 s /(2|\Theta|)}} \sum_{w \in \partial^{\circ} W_{x}} \mathbb{E}\left\{\left|G_{\omega, \Lambda \backslash W_{x}}(z ; x, w)\right|^{s /(2|\Theta|)}\right\}<b
$$

is satisfied for some $b \in(0,1)$, arbitrary $\Lambda \subset \Gamma$, and all $x \in \Lambda$, then for all $x, y \in \Gamma$

$$
\mathbb{E}\left\{\left|G_{\omega, \Gamma}(z ; x, y)\right|^{s /(2|\Theta|)}\right\} \leq A \mathrm{e}^{-\mu|x-y|_{\infty}} .
$$

Here

$$
A=\frac{C_{s} \Xi_{s}(\lambda)}{b} \quad \text { and } \quad \mu=\frac{|\ln b|}{L+\operatorname{diam} \Theta+2},
$$

with $C_{s}$ as in Lemma 7.2.

Note that condition (6) can be achieved by choosing $\lambda$ sufficiently big and applying Lemma 7.2. The core of the proof of the theorem is the following Lemma 7.2 ,

Lemma 7.4 ([ETV11]). Let Assumption $(A)$ be satisfied. Let $\Gamma \subset \mathbb{Z}^{d}$, $s \in(0,1 / 3), m>0$ and $b_{s}(\lambda, L, \Lambda)$ be the constant from Theorem 7.3. Then we have for all $x, y \in \Gamma$ with $y \notin \Lambda_{x}$ and all $z \in \mathbb{C} \backslash \mathbb{R}$ with $|z| \leq m$ the bound

$$
\mathbb{E}\left\{\left|G_{\omega, \Gamma}(z ; x, y)\right|^{\frac{s}{2|\Theta|}}\right\} \leq \frac{b_{s}(\lambda, L, \Gamma)}{\left|\partial^{\circ} \Lambda_{x}\right|} \sum_{r \in \partial^{\circ} \Lambda_{x}} \mathbb{E}\left\{\mid G_{\omega, \Gamma \backslash \Lambda_{x}}(z ; r, y)^{\frac{s}{|| \Theta \mid}}\right\} .
$$

The combination of Theorem 7.3 and Lemma 7.2 yield the following result on exponential decay of a fractional moment of the Green function under a strong disorder assumption.

Theorem 7.5 ([ETV11]). Let $\Gamma \subset \mathbb{Z}^{d}, s \in(0,1 / 3)$ and suppose that Assumption $(A)$ is satisfied. Then for a sufficiently large $\lambda$ there are constants $\mu, A \in \mathbb{R}^{+}$, depending only on $d, \rho, u, s$ and $\lambda$, such that for all $z \in \mathbb{C} \backslash \mathbb{R}$ and all $x, y \in \Gamma$

$$
\mathbb{E}\left\{\left|G_{\omega, \Gamma}(z ; x, y)\right|^{s /(2|\Theta|)}\right\} \leq A \mathrm{e}^{-\mu|x-y|_{\infty}} .
$$

7.3. Localization. The existing proofs of localization via the fractional moment method use either the Simon Wolff criterion, see e.g. SW86, AM93, ASFH01, or the RAGE-Theorem, see e.g. [Aiz94, Gra94, AEN ${ }^{+}$06]. Neither dynamical nor spectral localization can be directly inferred from the behavior of the Green function using the existent methods for the model in Section 2. The reason is that the random variables $V_{\omega}(x), x \in \mathbb{Z}^{d}$, are not independent, while the dependence of $H_{\omega}$ on the i.i.d. random variables $\omega_{k}$, $k \in \mathbb{Z}^{d}$, is not monotone.

We outline a new variant for concluding exponential localization from bounds on averaged fractional powers of Green's function (cf. Section 7.2) 
without using the multiscale analysis, see [ETV10, ETV11] for details. This is done by showing that fractional moment bounds imply the "typical output" of the multiscale analysis, i.e. the hypothesis of Theorem 2.3 in [DK89]. Then one can conclude localization using existent methods.

The next Proposition states that certain bounds on averaged fractional moments of Green's function imply the hypothesis of Theorem 2.3 in [DK89] (without applying the induction step of the multiscale analysis).

Proposition 7.6 ([ETV11] ). Let $I \subset \mathbb{R}$ be a bounded interval and $s \in(0,1)$. Assume the following two statements:

(i) There are constants $C, \mu \in \mathbb{R}^{+}$and $L_{0} \in \mathbb{N}_{0}$ such that

$$
\mathbb{E}\left\{\left|G_{\omega, \Lambda_{L, k}}(E ; x, y)\right|^{s}\right\} \leq C \mathrm{e}^{-\mu|x-y|_{\infty}}
$$

for all $k \in \mathbb{Z}^{d}, L \in \mathbb{N}, x, y \in \Lambda_{L, k}$ with $|x-y|_{\infty} \geq L_{0}$, and all $E \in I$.

(ii) There is a constant $C^{\prime} \in \mathbb{R}^{+}$such that

$$
\mathbb{E}\left\{\left|G_{\omega, \Lambda_{L, k}}(E+\mathrm{i} \varepsilon ; x, x)\right|^{s}\right\} \leq C^{\prime}
$$

for all $k \in \mathbb{Z}^{d}, L \in \mathbb{N}, x \in \Lambda_{L, k}, E \in I$ and all $\varepsilon \in(0,1]$.

Then we have for all $L \geq \max \left\{8 \ln (8) / \mu, L_{0},-(8 / 5 \mu) \ln (|I| / 2)\right\}$ and all $x, y \in \mathbb{Z}^{d}$ with $|x-y|_{\infty} \geq 2 L+\operatorname{diam} \Theta+1$ that

$$
\begin{aligned}
\mathbb{P}\left\{\forall E \in I \text { either } \Lambda_{L, x} \text { or } \Lambda_{L, y} \text { is }(\mu / 8, E) \text {-regular }\right\} \\
\qquad 1-8\left|\Lambda_{L, x}\right|\left(C|I|+4 C^{\prime}\left|\Lambda_{L, x}\right| / \pi\right) \mathrm{e}^{-\mu s L / 8} .
\end{aligned}
$$

In the proof of Proposition 7.6 Hypothesis (ii) is only used to obtain a Wegner estimate. In particular, there is a relation between a Wegner estimate and the a priori bound in the fractional moment method. The following proposition states that the boundedness of averaged fractional powers of the diagonal Green function elements implies a Wegner estimate.

Proposition 7.7 ([ETV11]). Let $I \subset \mathbb{R}$ be an interval, $s \in(0,1)$ and $c>0$. Assume there is a constant $C \in \mathbb{R}^{+}$such that

$$
\mathbb{E}\left\{\left|G_{\omega, L}(E+\mathrm{i} \varepsilon ; x, x)\right|^{s}\right\} \leq C
$$

for all $L \in \mathbb{N}, x \in \Lambda_{L}, E \in I$ and all $\varepsilon \in(0, c]$. Then we have for all $[a, b] \subset I$ with $0<b-a \leq c$ that

$$
\mathbb{E}\left\{\operatorname{Tr} \chi_{[a, b]}\left(H_{\omega, L}\right)\right\} \leq \frac{4 C}{\pi}|b-a|^{s}\left|\Lambda_{L}\right|
$$

Proof. Let $[a, b] \subset I$ with $0<b-a \leq c$. Since we have for any $\lambda \in \mathbb{R}$ and $0<\varepsilon \leq b-a$

$$
\arctan \left(\frac{\lambda-a}{\varepsilon}\right)-\arctan \left(\frac{\lambda-b}{\varepsilon}\right) \geq \frac{\pi}{4} \chi_{[a, b]}(\lambda),
$$

one obtains an inequality version of Stones formula:

$$
\left\langle\delta_{x}, \chi_{[a, b]}\left(H_{\omega, L}\right) \delta_{x}\right\rangle \leq \frac{4}{\pi} \int_{[a, b]} \operatorname{Im}\left\{G_{\omega, L}(E+\mathrm{i} \varepsilon ; x, x)\right\} \mathrm{d} E \quad \forall \varepsilon \in(0, b-a] .
$$


Using triangle inequality, $|\operatorname{Im} z| \leq|z|$ for $z \in \mathbb{C}$, Fubini's theorem, $\mid G_{\omega, L}(E+$ i $\varepsilon ; x, x)\left.\right|^{1-s} \leq \operatorname{dist}\left(\sigma\left(H_{\omega, L}\right), E+i \varepsilon\right)^{s-1} \leq \varepsilon^{s-1}$ and hypothesis (ii) we obtain for all $\varepsilon \in(0, b-a]$

$$
\begin{aligned}
\mathbb{E}\left\{\operatorname{Tr} \chi_{[a, b]}\left(H_{\omega, L}\right)\right\} & \leq \mathbb{E}\left\{\sum_{x \in \Lambda_{L}} \frac{4}{\pi} \int_{[a, b]} \operatorname{Im}\left\{G_{\omega, L}(E+\mathrm{i} \varepsilon ; x, x)\right\} \mathrm{d} E\right\} \\
& \leq \frac{\varepsilon^{s-1}}{\pi / 4} \sum_{x \in \Lambda_{L}} \int_{[a, b]} \mathbb{E}\left\{\left|G_{\omega, L}(E+\mathrm{i} \varepsilon ; x, x)\right|^{s}\right\} \mathrm{d} E \\
& \leq 4 \pi^{-1} \varepsilon^{s-1}\left|\Lambda_{L}\right||b-a| C .
\end{aligned}
$$

We minimize the right hand side by choosing $\varepsilon=b-a$ and obtain the statement of the proposition.

Let us note that a Wegner estimate implies the boundedness of an averaged fractional power of the (finite-volume) Green function. At the moment we only know a proof where the bound depends polynomially on the volume of the cube.

From the discussion so far it follows that Hypothesis (ii) of Proposition 7.6 can be replaced by a Wegner estimate. Specifically, the following assertion holds true.

Proposition 7.8 ([ETV11] $)$. Let $I \subset \mathbb{R}$ be a bounded interval and $s \in(0,1)$. Assume the following two statements:

(i) There are constants $C, \mu \in \mathbb{R}^{+}$and $L_{0} \in \mathbb{N}_{0}$ such that

$$
\mathbb{E}\left\{\left|G_{\omega, \Lambda_{L, k}}(E ; x, y)\right|^{s}\right\} \leq C \mathrm{e}^{-\mu|x-y|_{\infty}}
$$

for all $k \in \mathbb{Z}^{d}, L \in \mathbb{N}, x, y \in \Lambda_{L, k}$ with $|x-y|_{\infty} \geq L_{0}$, and all $E \in I$.

(ii) There are constants $C_{\mathrm{W}} \in \mathbb{R}^{+}, \beta \in(0,1]$, and $D \in \mathbb{N}$ such that

$$
\mathbb{P}\left\{\sigma\left(H_{\omega, \Lambda_{L}}\right) \cap[a, b] \neq \emptyset\right\} \leq C_{\mathrm{W}}|b-a|^{\beta} L^{D}
$$

for all $L \in \mathbb{N}$ and all $[a, b] \subset I$.

Then we have for all $L \geq \max \left\{8 \ln (2) / \mu, L_{0},-(8 / 5 \mu) \ln (|I| / 2)\right\}$ and all $x, y \in \mathbb{Z}$ with $|x-y|_{\infty} \geq 2 L+\operatorname{diam} \Theta+1$ that

$$
\begin{aligned}
\mathbb{P}\left\{\forall E \in I \text { either } \Lambda_{L, x} \text { or } \Lambda_{L, y}\right. & \text { is }(\mu / 8, E) \text {-regular }\} \\
& \geq 1-8(2 L+1)^{d} \mid\left(C|I|+C_{\mathrm{W}} L^{D}\right) \mathrm{e}^{-\mu \beta L / 8} .
\end{aligned}
$$

To conclude exponential localization from the estimates provided in Proposition 7.6 or 7.8 we will use Theorem 2.3 in [DK89]. More precisely we need a slight extension of the result, which can be proven with the same arguments as the original result. What matters for the proof of Theorem 7.9 is that there is an $l_{0} \in \mathbb{N}$ such that potential values at different lattice sites are independent if their distance is larger or equal $l_{0}$. 
Theorem 7.9 ([DK89]). Let $I \subset \mathbb{R}$ be an interval and let $p>d, L_{0}>1$, $\alpha \in(1,2 p / d)$ and $m>0$. Set $L_{k}=L_{k-1}^{\alpha}$, for $k \in \mathbb{N}$. Suppose that for any $k \in \mathbb{N}_{0}$

$$
\mathbb{P}\left\{\forall E \in I \text { either } \Lambda_{L_{k}, x} \text { or } \Lambda_{L_{k}, y} \text { is }(m, E) \text {-regular }\right\} \geq 1-L_{k}^{-2 p}
$$

for any $x, y \in \mathbb{Z}^{d}$ with $|x-y|_{\infty} \geq 2 L_{k}+\operatorname{diam} \Theta+1$. Then $H_{\omega}$ exhibits exponential localization in $I$ for almost all $\omega \in \Omega$.

From Proposition 7.6 and Theorem 7.9 we conclude that the decay of fractional moments of the Green function implies exponential localization.

Theorem 7.10 (ETV11]). Let $s \in(0,1), C, \mu, \in \mathbb{R}^{+}$, and $I \subset \mathbb{R}$ be a interval. Assume that

$$
\mathbb{E}\left\{\left|G_{\omega, \Lambda_{L, k}}(E+\mathrm{i} \varepsilon ; x, y)\right|^{s}\right\} \leq C \mathrm{e}^{-\mu|x-y|_{\infty}}
$$

for all $k \in \mathbb{Z}^{d}, L \in \mathbb{N}, x, y \in \Lambda_{L, k}, E \in I$ and all $\varepsilon \in(0,1]$. Then $H_{\omega}$ exhibits exponential localization in I for almost all $\omega \in \Omega$.

Putting together Theorem 7.5 and Theorem 7.10, one can prove without the use of MSA exponential localization in the case of sufficiently large disorder.

Theorem 7.11 ([ETV11], Exponential localization via fractional moments). Let Assumption $(A)$ be satisfied and $\lambda$ sufficiently large. Then $H_{\omega}$ exhibits exponential localization for almost all $\omega \in \Omega$.

\section{Localization Via mUltiscale ANALYSis}

The goal of this section is to explain how to obtain localization for the discrete alloy-type model using multiscale analysis under the assumptions

- $u(0) \neq 0,|u(k)| \leq \mathrm{e}^{-c|k|_{\infty}}$ for some positive constant $c$, and

- the measure $\mu$ has a bounded density $\rho$ with $\operatorname{supp} \rho \subset[-1,1]$.

For background on multiscale analysis, we refer for example to [Kir08] and [Sto01. The main idea of multiscale analysis is to show that Green's function decay on a small scale implies Green's function decay on a larger scale. In order to quantify decay of the Green's function, we introduce

Definition 8.1. Let $\gamma>0$. A box $\Lambda_{r, n} \subseteq \mathbb{Z}^{d}$ is called $\gamma$-suitable for $H_{\omega}-E$ if

(i) $\left\|\left(H_{\omega, \Lambda_{r, n}}-E\right)^{-1}\right\| \leq \mathrm{e}^{\sqrt{r}}$.

(ii) For $x, y \in \Lambda_{r, n}$ with $|x-y| \geq \frac{r}{10}$, we have

$$
\left|G_{\omega, \Lambda_{r, n}}(E ; x, y)\right| \leq \frac{1}{\#\left(\Lambda_{r, n}\right)} \mathrm{e}^{-\gamma|x-y|} .
$$

Only condition (ii) is needed for multiscale analysis when a Wegner estimate is available. However, our goal is to do without it and then (i) takes the role of the Wegner estimate.

The essential step in Krü] to conclude exponential and dynamical localization is 
Theorem 8.2. Given $\gamma>0$ and $E \in \mathbb{R}$, there is $R=R(\gamma, u, \mu) \geq 1$ such that if

$$
\mathbb{P}\left\{\Lambda_{r} \text { is } \gamma \text {-suitable for } H_{\omega}-E\right\} \leq \frac{1}{r^{4 d}}
$$

holds for $1 \leq r \leq R$ then it holds for all $r>0$ with $\gamma$ replaced by $\gamma / 2$.

This theorem implies localization at large coupling:

- For large coupling, the Combes-Thomas estimate and a probabilistic computation imply the assumptions of Theorem 8.2 . This can be found in Appendix A of [Krü].

- The conclusions of this theorem imply localization. Exponential localization is proven in Section 7 of [BK05] and dynamical localization in Sections 16 to 18 of Krü].

Theorem 8.3 ([Krü] $)$. Let $\lambda>0$ be large enough. Then, for almost all $\omega \in \Omega, H_{\omega}$ exhibits dynamical (and spectral) localization.

We will now discuss how to prove Theorem 8.2 . The main difficulty is to conclude from a probabilistic estimate on a small scale, i.e. (17) for some $r \geq 1$, the estimate on the resolvent with a better probability, i.e.

$$
\mathbb{P}\left\{\left\|\left(H_{\omega, R}-E\right)^{-1}\right\|>\mathrm{e}^{\sqrt{R}}\right\}<\frac{1}{R^{4 d}} .
$$

It turns out, that we do not know how to do this. Instead we assume that (7) holds for all values of $r$ in a range $\left[r_{1}, r_{2}\right]$ with $r_{2}=\left(r_{1}\right)^{3}$ and use this to conclude (8).

Just using a single value of $r$ one can conclude that there exists an event $\mathcal{B}$ with $\mathbb{P}\{\mathcal{B}\} \leq 1 /\left(2 R^{4 d}\right)$, such that for $\omega \notin \mathcal{B}$, there are $m_{1}, \ldots, m_{L}$ with $L$ uniformly bounded in $R$ such that for

$$
\Xi=\Lambda_{R} \backslash \bigcup_{\ell=1}^{L} \Lambda_{r, m_{\ell}}
$$

we have

$$
\left\|\left(H_{\omega, \Xi}-E\right)^{-1}\right\| \leq \mathrm{e}^{3 \sqrt{r}} .
$$

This follows from the probabilistic estimates in Section 5 and the estimates on the resolvent in Section 9 of [Krü].

Then one uses the assumption on the whole range $\left[r_{1}, r_{2}\right]$ to conclude that there exists a choice $\tilde{\omega}$ which agrees with $\omega$ except near the $m_{\ell}$ such that

$$
\left\|\left(H_{\tilde{\omega}, R}-E\right)^{-1}\right\| \leq \mathrm{e}^{10 \sqrt{r_{2}}} .
$$

We illustrate this in the next subsection. See Section 12 of [Krü] or Section 2 in [Bou09] for the entire implementation.

In Subsection 8.2, we illustrate how to use Cartan's lemma to conclude from this information that the estimate (8) holds. For the entire analysis, see Sections 13 to 15 in [Krï]. 
Then one can conclude the decay of the Green function as in the usual multiscale analysis finishing the proof of Theorem 8.2 .

8.1. Probabilistic estimates. In this section, we illustrate a new form of probabilistic estimate not necessary in the usual versions of multiscale analysis. We will assume for $r_{\ell}=r \cdot \ell$ with $\ell=1, \ldots, L$ that

$$
\mathbb{P}\left\{\left\|\left(H_{\omega, r_{\ell}}-E\right)^{-1}\right\|>A\right\} \leq \varepsilon
$$

and that $\operatorname{supp}(u) \subseteq \Lambda_{r-1}$. The main conclusion will be

Lemma 8.4. There exists an event $\mathcal{B}$ with

$$
\mathbb{P}\{\mathcal{B}\} \leq \varepsilon^{L}
$$

such that for $\omega \notin \mathcal{B}$, there exists $\ell$ such that there exists $\tilde{\omega}$ with

$$
\omega_{n}=\tilde{\omega}_{n}, \quad n \notin \Lambda_{r_{\ell-1}}
$$

we have

$$
\left\|\left(H_{\tilde{\omega}, r_{\ell}}-E\right)^{-1}\right\| \leq A .
$$

Proof. Denote by $X_{\ell}$ the set of all $\omega$ such that for all $\tilde{\omega}$ with

$$
\omega_{n}=\tilde{\omega}_{n}, \quad n \notin \Lambda_{r_{\ell-1}}
$$

we have

$$
\left\|\left(H_{\tilde{\omega}, r_{\ell}}-E\right)^{-1}\right\|>A .
$$

By assumption, we have that $\mathbb{P}\left\{X_{\ell}\right\} \leq \varepsilon$ and one can check that $X_{\ell}$ and $X_{j}$ are independent events for $j \neq \ell$.

Take $\mathcal{B}=\bigcup_{\ell=1}^{L} X_{\ell}$. Independence implies $\mathbb{P}\{\mathcal{B}\} \leq \varepsilon^{L}$. The claim follows by construction.

This lemma implies that given $m \in \Lambda_{R}$, one can always find some $\tilde{r} \leq r^{2}$ and $\tilde{\omega}$ the resolvent estimate holds for the cube $\Lambda_{\tilde{r}, m}$. Using Theorem 9.4. in [Krü], one can then extend (9) to the estimate

$$
\left\|\left(H_{\tilde{\omega}, R}-E\right)^{-1}\right\| \leq \mathrm{e}^{r}
$$

for some $\tilde{\omega}$ for which $\tilde{\omega}_{n}=\omega_{n}$ whenever $\left|n-m_{\ell}\right| \geq r^{2}$.

8.2. An application of Cartan's lemma. In this section, we illustrate the application of Cartan's Lemma with a simple application to random Schrödinger operators. The main goal is to give a simplified account of what happens in Sections 11 to 14 of [Krü].

Suppose we want to establish a bound on $\left\|\left(H_{\omega, L}-E\right)^{-1}\right\|$ and that we already know

(1) For each $\omega$, there exists $n \in \Lambda_{L}$ such that

$$
\left\|\left(H_{\omega, \Lambda_{L} \backslash \Lambda_{r, n}}-E\right)^{-1}\right\| \leq A .
$$


(2) For each $n$ and $\omega$, there exists $\tilde{\omega}$ with

$$
\omega_{m}=\tilde{\omega}_{m}, \quad m \in \Lambda_{L} \backslash\{n\}
$$

and

$$
\left\|\left(H_{\tilde{\omega}, L}-E\right)^{-1}\right\| \leq A .
$$

This is a simplification: First, one must allow for more exceptional sites. Second, such a statement can allow hold in probabilistic terms in a multiscale scheme. Bou09] was the first paper to propose a scheme to check (2).

For simplicity, we will also assume that

(3) $\operatorname{supp}(u)$ is contained in $\Lambda_{r}$.

The analysis of $u$ exponential decaying requires a perturbative analysis, which we avoid here for the sake of exposition.

Proposition 8.5. Assume (1), (2), and (3). Then

$$
\mathbb{P}\left\{\left\|\left(H_{\omega, L}-E\right)^{-1}\right\| \geq \mathrm{e}^{s}\right\} \leq \mathrm{e}^{-\frac{\delta s}{\log (A) \cdot r d}}
$$

for some small constant $\delta>0$.

The proof of this proposition will be split into two parts. First, we consider the case when the single-site potential $u$ is equal to $\delta_{0}$. Second, we discuss what needs to be modified for general $u$. We can fix $n$. Then the claim follows by summing over the possible number of choices of $n$ (less than $(3 L)^{d}$ many).

Assume now that $u=\delta_{0}$, then we can write $H_{\omega, L}-E$ as

$$
H_{\omega, L}-E=\left(\begin{array}{cc}
\omega_{n}-E & \Gamma \\
\Gamma^{*} & H_{\omega, \Lambda_{L} \backslash\{n\}}-E
\end{array}\right) .
$$

An application of the Schur-complement formula shows that

$$
\left\|\left(H_{\omega, L}-E\right)^{-1}\right\| \leq C \cdot A^{2}\left|\omega_{n}-E-\Gamma\left(H_{\omega, \Lambda_{L} \backslash\{n\}}-E\right)^{-1} \Gamma^{*}\right|^{-1},
$$

for some constant $C$. Since the dependence in $\omega_{n}$ is linear, it is easy to see that the set of $\omega_{n}$, where the right hand side is small, is small. Hence, we are done.

It is noteworthy that this argument did not use (2). Of course, (2) and not even (1) is necessary since the standard proof of Wegner's estimate works.

Let us now discuss what happens when $u$ is not $\delta_{0}$, but is supported on finitely many points. One could apply the Schur-complement formula as before, but the object one then obtains has a too non-trivial dependence on $\omega_{n}$ to be useful. Define

$$
\tilde{\Xi}=\Lambda_{r, n} \cap \Lambda_{L}, \quad \tilde{\Theta}=\Lambda_{L} \backslash \tilde{\Xi} .
$$

Then $H_{\omega}^{\tilde{\Theta}}-E$ is independent of $\omega_{n}$. So an application of the Schur-complement formula shows that

$$
\left\|\left(H_{\omega, L}-E\right)^{-1}\right\| \leq C A^{2}\left\|\left(H_{\omega, \tilde{\Xi}}-E-\Gamma\left(H_{\omega, \tilde{\Theta}}-E\right)^{-1} \Gamma^{*}\right)^{-1}\right\| .
$$


Fix $\left\{\omega_{m}\right\}_{m \neq n}$ and define a function of single-variable $\omega_{n}$ by

$$
f\left(\omega_{n}\right)=\operatorname{det}\left(H_{\omega, \tilde{\Xi}}-E-\Gamma\left(H_{\omega, \tilde{\Theta}}-E\right)^{-1} \Gamma^{*}\right) .
$$

Define $R=\# \tilde{\Xi}$. By (1), we obtain that

$$
|f(x)| \leq C A^{R}
$$

and by (2) that

$$
\left|f\left(\tilde{\omega}_{n}\right)\right| \geq \frac{1}{A^{R}} .
$$

Hence, we can apply Cartan's Lemma to obtain

$$
\left|\left\{\omega_{n}: \quad\left|f\left(\omega_{n}\right)\right| \leq \mathrm{e}^{-s}\right\}\right| \leq C \exp \left(-\frac{s}{R \log (A)}\right) .
$$

The claim follows by $R \leq(3 r)^{d}$.

\section{REFERENCES}

$\left[\mathrm{AEN}^{+}\right.$06] M. Aizenman, A. Elgart, S. Naboko, J. H. Schenker, and G. Stolz, Moment analysis for localization in random Schrödinger operators, Invent. Math. 163 (2006), no. 2, 343-413.

[Aiz94] M. Aizenman, Localization at weak disorder: some elementary bounds, Rev. Math. Phys. 6 (1994), no. 5a, 1163-1182.

[AM93] M. Aizenman and S. Molchanov, Localization at large disorder and at extreme energies: An elementary derivation, Commun. Math. Phys. 157 (1993), no. 2, 245-278.

[ASFH01] M. Aizenman, J. H. Schenker, R. M. Friedrich, and D. Hundertmark, Finitevolume fractional-moment criteria for Anderson localization, Commun. Math. Phys. 224 (2001), no. 1, 219-253.

[BGS02] J. Bourgain, M. Goldstein, and W. Schlag, Anderson localization for Schrödinger operators on $\mathbb{Z}^{2}$ with quasi-periodic potential, Acta Math. 188 (2002), no. 1, 41-86.

[BK05] J. Bourgain and C. E. Kenig, On localization in the continuous AndersonBernoulli model in higher dimension, Invent. Math. 161 (2005), no. 2, 389426.

[BLS08] J. Baker, M. Loss, and G. Stolz, Minimizing the ground state energy of an electron in a randomly deformed lattice, Commun. Math. Phys. 283 (2008), no. 2, 397-415.

[Bou04] J. Bourgain, Recent progress in quasi-periodic lattice Schrödinger operators and Hamiltonian partial differential equations, Uspekhi Mat. Nauk 59 (2004), no. 2(356), 37-52.

[Bou07] _ Anderson localization for quasi-periodic lattice Schrödinger operators on $\mathbb{Z}^{d}$, d arbitrary, Geom. Funct. Anal. 17 (2007), no. 3, 682-706.

[Bou09] _ An approach to Wegner's estimate using subharmonicity, J. Stat. Phys 134 (2009), no. 5-6, 969-978.

[CKM87] R. Carmona, A. Klein, and F. Martinelli, Anderson localization for Bernoulli and other singular potentials, Commun. Math. Phys. 108 (1987), no. 1, 41-66.

[DK89] H. von Dreifus and A. Klein, A new proof of localization in the Anderson tight binding model, Commun. Math. Phys. 124 (1989), no. 2, 285-299.

[DK91] L L L L Lalization for random Schrödinger operators with correlated potentials, Commun. Math. Phys. 140 (1991), no. 1, 133-147. 
[dRJLS96] R. del Rio, S. Jitomirskaya, Y. Last, and B. Simon, Operators with singular continuous spectrum, IV. Hausdorff dimensions, rank one perturbations, and localization, J. Anal. Math. 69 (1996), no. 1, 153-200.

[DS87] R. L. Dobrushin and S. Shlosman, Completely analytical interactions: Constructive description, J. Stat. Phys. 46 (1987), no. 5-6, 983-1014.

[EHa] L. Erdős and D. Hasler, Anderson localization at band edges for random magnetic fields, arXiv:1103.3744 1 [math-ph].

[EHb] Anderson localization for random magnetic Laplacian on $\mathbb{Z}^{2}$, arXiv:1101.2139v1 [math-ph].

[EHc] Wegner estimate and Anderson localization for random magnetic fields, to appear in Commun. Math. Phys, arXiv:1012.5185 1 [math-ph].

[ETV10] A. Elgart, M. Tautenhahn, and I. Veselić, Localization via fractional moments for models on $\mathbb{Z}$ with single-site potentials of finite support, J. Phys. A: Math. Theor. 43 (2010), no. 47, 474021.

[ETV11] _ Anderson localization for a class of models with a sign-indefinite single-site potential via fractional moment method, Ann. Henri Poincaré (2011), DOI: 10.1007/s00023-011-0112-5.

[FS83] J. Fröhlich and T. Spencer, Absence of diffusion in the Anderson tight binding model for large disorder or low energy, Commun. Math. Phys. 88 (1983), no. 2, 151-184.

[GK04] F. Germinet and A. Klein, A characterization of the Anderson metal-insulator transport transition, Duke Math. J. 124 (2004), no. 2, 309-350.

[Gra94] G. M. Graf, Anderson localization and the space-time characteristic of continuum states, J. Stat. Phys. 75 (1994), no. 1-2, 337-346.

[HK02] P. D. Hislop and F. Klopp, The integrated density of states for some random operators with nonsign definite potentials, J. Funct. Anal. 195 (2002), no. 1, $12-47$.

[HLMW01] T. Hupfer, H. Leschke, P. Müller, and S. Warzel, The absolute continuity of the integrated density of states for magnetic Schrödinger operators with certain unbounded random potentials, Commun. Math. Phys. 221 (2001), no. 2, 229254.

[Kir89] W. Kirsch, Random Schrödinger operators, Schrödinger Operators (Berlin) (H. Holden and A. Jensen, eds.), Lecture Notes in Physics, 345, Springer, 1989.

[Kir08] W. Kirsch, An invitation to random Schrödinger operators, Random Schrödinger operators, Panor. Synthèses, vol. 25, Soc. Math. France, 2008, With an appendix by Frédéric Klopp, pp. 1-119.

[KLNS] F. Klopp, M. Loss, S. Nakamura, and G. Stolz, Localization for the random displacement model, arXiv:1007.2483v2 [math-ph].

[Klo93] F. Klopp, Localization for semiclassical continuous random Schrödinger operators II: The random displacement model, Helv. Phys. Acta 66 (1993), no. 7-8, 810-841.

[Klo95] F. Klopp, Localization for some continuous random Schrödinger operators, Commun. Math. Phys. 167 (1995), no. 3, 553-569.

[Klo02] F. Klopp, Weak disorder localization and Lifshitz tails: continuous Hamiltonians, Ann. Henri Poincaré 3 (2002), no. 4, 711-737.

[KM82] W. Kirsch and F. Martinelli, On the spectrum of Schrödinger operators with a random potential, Commun. Math. Phys. 85 (1982), no. 3, 329-350.

[KM07] W. Kirsch and B. Metzger, The integrated density of states for random Schrödinger operators, Spectral Theory and Mathematical Physics, Proceedings of Symposia in Pure Mathematics, vol. 76, AMS, 2007, pp. 649-698. 
[KN09] F. Klopp and S. Nakamura, Spectral extrema and Lifshitz tails for nonmonotonous alloy type models, Commun. Math. Phys. 287 (2009), no. 3, 1133-1143.

[KNNN03] F. Klopp, S. Nakamura, F. Nakano, and Y. Nomura, Anderson localization for $2 D$ discrete Schrödinger operators with random magnetic fields, Ann. Henri Poincaré 4 (2003), no. 4, 795-811.

[Krü] H. Krüger, Localization for random operators with non-monotone potentials with exponentially decaying correlations, to appear in Ann. Henri Poincaré, DOI: 10.1007/s00023-011-0130-3, arXiv:1006.5233v1 [math.SP].

[KSS98a] W. Kirsch, P. Stollmann, , and G. Stolz, Anderson localization for random Schrödinger operators with long range interactions, Commun. Math. Phys. 195 (1998), no. 3, 495-507.

[KSS98b] W. Kirsch, P. Stollmann, and G. Stolz, Localization for random perturbations of periodic Schrödinger operators, Random Oper. Stochastic Equations 6 (1998), no. 3, 241-268.

[KV06] V. Kostrykin and I. Veselić, On the Lipschitz continuity of the integrated density of states for sign-indefinite potentials, Math. Z. 252 (2006), no. 2, 367-392.

[Lev96] B. Ya. Levin, Lectures on entire functions, Translations of Mathematical Monographs, no. 150, American Mathematical Society, 1996.

[LPPV08] D. Lenz, N. Peyerimhoff, O. Post, and I. Veselić, Continuity properties of the integrated density of states on manifolds, Jpn. J. Math. 3 (2008), no. 1, $121-161$.

[LPPV09] _ Continuity of the integrated density of states on random length metric graphs, Math. Phys. Anal. Geom. 12 (2009), no. 3, 219-254.

[LPV04] D. Lenz, N. Peyerimhoff, and I. Veselić, Integrated density of states for random metrics on manifolds, Proc. London Math. Soc. (3) 88 (2004), no. 3, 733-752.

[NSV03] F. Nazarov, M. Sodin, and A. Volberg, Local dimension-free estimates for volumes of sublevel sets of analytic functions, Isr. J. Math. 133 (2003), no. 1, 269-283.

[PTV11] N. Peyerimhoff, M. Tautenhahn, and I. Veselić, Wegner estimate for alloy-type models with sign-changing and exponentially decaying single-site potentials, TU Chemnitz Preprint 9, June 2011.

[Sto00] G. Stolz, Non-monotonic random Schrödinger operators: the Anderson model, J. Math. Anal. Appl. 248 (2000), no. 1, 173-183.

[Sto01] P. Stollmann, Caught by disorder: Bound states in random media, Progress in Mathematical Physics, vol. 20, Birkhäuser, 2001.

[Sto10] G. Stolz, An introduction to the mathematics of Anderson localization, Lecture notes of the Arizona School of Analysis with Applications, 2010, to appear in Contemp. Math., arXiv:1104.2317 v 1 [math-ph].

[SW86] B. Simon and T. Wolff, Singular continuous spectrum under rank one perturbations and localization for random Hamiltonians, Commun. Pur. Appl. Math. 39 (1986), no. 1, 75-90.

[TV10] M. Tautenhahn and I. Veselić, Spectral properties of discrete alloy-type models, Proceedings of the XVIth International Congress on Mathematical Physics, Prague, 2009, World Scientific, 2010.

[Uek94] N. Ueki, On spectra of random Schrödinger operators with magnetic fields, Osaka J. Math. 31 (1994), no. 1, 177-187.

[Uek00] _ Simple examples of Lifschitz tails in Gaussian random magnetic fields, Ann. Henri Poincaré 1 (2000), no. 3, 473-498.

[Uek04] Wegner estimates and localization for Gaussian random potentials, Publ. Res. Inst. Math. Sci. 40 (2004), no. 1, 29-90. 
[Uek08] — Wegner estimate and localization for random magnetic fields, Osaka J. Math. 45 (2008), no. 3, 565-608.

[Ves02] I. Veselić, Wegner estimate and the density of states of some indefinite alloy type Schrödinger operators, Lett. Math. Phys. 59 (2002), no. 3, 199-214.

[Ves07] I. Veselić, Existence and regularity properties of the integrated density of states of random Schrödinger Operators, Lecture Notes in Mathematics, vol. Vol. 1917, Springer-Verlag, 2007.

[Ves10a] I. Veselić, Wegner estimate for discrete alloy-type models, Ann. Henri Poincaré 11 (2010), no. 5, 991-1005.

[Ves10b] I. Veselić, Wegner estimates for sign-changing single site potentials, Math. Phys. Anal. Geom. 13 (2010), no. 4, 299-313.

[Ves11] I. Veselić, Lipschitz-continuity of the integrated density of states for Gaussian random potentials, Lett. Math. Phys. 97 (2011), no. 1.

[Weg81] F. Wegner, Bounds on the DOS in disordered systems, Z. Phys. B 44 (1981), no. 1-2, 9-15.

448 Department of Mathematics, McBryde Hall, Virginia Tech., BlacksBURG, VA, 24061, USA

URL: http://www.math.vt.edu/people/aelgart/

Caltech Department of Mathematics, Mailcode 253-37, 1200 E California Blvd, Pasadena CA 91125, USA

URL: http://www.its.caltech.edu/ helge/

Technische Universität Chemnitz, Fakultät für Mathematik, D-09107 ChemNITZ, GERMANY

URL: http://www-user.tu-chemnitz.de/ ${ }^{\mathrm{mtau} /}$

Technische Universität Chemnitz, FAkultät Für Mathematik, D-09107 ChemNitZ, Germany

$U R L:$ http://www.tu-chemnitz.de/mathematik/stochastik/ 